\title{
Comparações entre os conjuntos de soluções de Carathéodory e de Sentis
}

\section{Comparison between the Carathéodory and Sentis solution sets}

\author{
Iguer Luis Domini dos Santos \\ Universidade Estadual Paulista (UNESP), Departamento de Matemática, Ilha Solteira, SP, Brasil \\ http://orcid.org/0000-0001-5237-6024, iguer.santos@unesp.br
}

\section{Informações do Artigo}

(c)

Histórico do artigo

Submissão: 31 de março de 2019.

Aceite: 20 de maio de 2019.

\section{Palavras-chave}

Equações Diferenciais Descontínuas

Soluções Generalizadas

Solução de Carathéodory

Solução de Sentis

\begin{abstract}
Resumo
No presente trabalho são estudadas as soluções generalizadas de Carathéodory e de Sentis para equações diferenciais descontínuas. Dessa forma, são estudadas relações de inclusão entre os conjuntos de soluções de Carathéodory e de Sentis. A partir de resultados da literatura, são estabelecidos aqui resultados análogos para relações entre as soluções de Carathéodory e de Sentis. Assim, estabelecendo analogias com resultados da literatura, são obtidas aqui comparações por meio de relações de inclusão entre os conjuntos de soluções de Carathéodory e de Sentis.
\end{abstract}

\section{Keywords}

Discontinuous Differential Equations

Generalized Solutions

Carathéodory Solution

Sentis Solution

\begin{abstract}
In the present work the Carathéodory and Sentis generalized solutions to discontinuous differential equations are studied. In this way, inclusion relations between the Carathéodory and Sentis solution sets are studied. From results of the literature, analogous results are established here for relations between the Carathéodory and Sentis solutions. Thus, by establishing analogies with literature results, comparisons are obtained here by means of inclusion relations between the Carathéodory and Sentis solution sets.
\end{abstract}

\section{Introdução}

Neste trabalho são estudadas equações diferenciais ordinárias com lados direitos descontínuos, também chamadas de equações diferenciais descontínuas. Sendo assim, estuda-se aqui soluções generalizadas para o problema de valor inicial

$$
\dot{x}(t)=f(x(t)), x(0)=x_{0}
$$

onde $t \in[0, T]$ e $f: \mathbb{R}^{n} \rightarrow \mathbb{R}^{n}$. O problema de valor inicial[1 foi abordado por Hu (1991) e por Spraker e Biles (1996) para o estudo das soluções generalizadas de Carathéodory, Filippov e de Krasovskii. Spraker e Biles (1996) abordam relações entre os conjuntos de soluções de Carathéodory e de Filippov, já Hu (1991) aborda relações entre os conjuntos de soluções de Carathéodory e de Krasovskii. A 
comparação por meio da relação de inclusão entre conjuntos de soluções generalizadas, também foi abordada por Bacciotti (2004), Hajek (1979) e por Sentis (1978). Dessa forma, por meio de exemplos não-triviais Bacciotti (2004) mostra que soluções de Sentis, soluções de Forward-Euler e soluções de Carathéodory são noções independentes. Já Hajek (1979) estuda e compara soluções de Filippov com outras noções de soluções, entre elas as noções devidas a Krasovskii, Hermes, Newton e Carathéodory. Por fim, Sentis (1978) introduz e compara as soluções de Sentis com as soluções de Filippov. O conceito de soluções de Sentis foi proposto por Bacciotti (2005). Como observado por Bacciotti (2004), na literatura ainda não foram completamente compreendidas as relações entre todas essas noções de soluções. Assim, motivados por Bacciotti (2004) nós estudamos relações de inclusão entre os conjuntos de soluções de Carathéodory e de Sentis do problema de valor inicial 1. Portanto, se $\mathcal{C}\left(f, x_{0}\right)$ e $\mathcal{S}\left(f, x_{0}\right)$ denotam os conjuntos de soluções de Carathéodory e de Sentis do problema de valor inicial 11, respectivamente, então serão estudadas relações de inclusão entre os conjuntos $\mathcal{C}\left(f, x_{0}\right)$ e $\mathcal{S}\left(f, x_{0}\right)$. As principais contribuições do trabalho se encontram no Teorema 4.1 . Teorema 4.5e Teorema 4.10.

\section{Preliminares}

A seguir são considerados conceitos e resultados básicos que são utilizados ao longo do presente trabalho.

\subsection{Lebesgue Mensurabilidade}

Aqui são relembrados conceitos básicos da teoria da medida. Uma abordagem mais completa a medida e integral de Lebesgue, pode ser encontrada em Royden (1963).

Definição 2.1. Uma função $f: \mathbb{R}^{n} \rightarrow \mathbb{R}^{n}$ é chamada de Lebesgue mensurável se para todo conjunto aberto $V \subset \mathbb{R}^{n}$, o conjunto

$$
f^{-1}(V)=\left\{x \in \mathbb{R}^{n}: f(x) \in V\right\}
$$

for Lebesgue mensurável.

Seja $I \subset \mathbb{R}$ um intervalo. Diz-se que uma proposição $P$ vale em quase todo ponto (q.t.p.) de $I$, se o conjunto $N$ dado por

$$
N=\{t \in I: P \text { não vale em } t\}
$$

tiver medida de Lebesgue zero. 


\subsection{Funções Absolutamente Contínuas}

Abaixo definimos funções absolutamente contínuas. A definição de função absolutamente contínua também pode ser encontrada em Aubin e Cellina (1984) e Smirnov (2002).

Definição 2.2. Uma função $x:[a, b] \rightarrow \mathbb{R}^{n}$ é dita absolutamente contínua se, dado $\varepsilon>0$, existe $\delta>0$ tal que para quaisquer coleção enumerável de subintervalos disjuntos $\left[a_{k}, b_{k}\right]$ de $[a, b]$ satisfazendo

$$
\sum\left(b_{k}-a_{k}\right)<\delta
$$

temos

$$
\sum\left|x\left(b_{k}\right)-x\left(a_{k}\right)\right|<\varepsilon
$$

Enunciamos a seguir um resultado considerado em Aubin e Cellina (1984) para funções absolutamente contínuas.

Teorema 2.3. Uma função contínua é a integral de sua derivada se e somente se ela é uma função absolutamente contínua.

Como abordado em Smirnov (2002), uma função absolutamente contínua $x:[a, b] \rightarrow \mathbb{R}^{n}$ é diferenciável em quase todo ponto de $[a, b]$, e sua derivada $\dot{x}(\cdot)$ é uma função Lebesgue integrável. Além disso, vale a fórmula de Newton-Leibniz; ou seja,

$$
x\left(t_{2}\right)-x\left(t_{1}\right)=\int_{t_{1}}^{t_{2}} \dot{x}(t) d t
$$

para todo $t_{1}, t_{2} \in[a, b], t_{1}<t_{2}$. Assim, qualquer função absolutamente contínua $x:[a, b] \rightarrow \mathbb{R}^{n}$ pode ser representada na forma

$$
x(t)=x(a)+\int_{a}^{t} \dot{x}(s) d s .
$$

\section{Soluções Generalizadas}

Nesta seção são introduzidas as soluções generalizadas de Carathéodory, Krasovskii, Filippov e de Sentis do problema de valor inicial 1 .

\subsection{Soluções de Carathéodory}

Definição 3.1. Uma função absolutamente contínua $x:[0, T] \rightarrow \mathbb{R}^{n}$ é uma solução de Carathéodory para o problema de valor inicial 1 se, e somente se, $x$ satisfizer a equação diferencial dada na equação 1 para q.t.p. $t \in[0, T]$ e $x(0)=x_{0}$. 
Soluções de Carathéodory são tratadas, por exemplo, por Coddington e Levinson (1955), Hale (1980), Hu (1991) e por Spraker e Biles (1996). A seguir abordamos a existência de soluções de Carathéodory de acordo com Coddington e Levinson (1955). Dessa forma, seja $f: D \subset \mathbb{R}^{n+1} \rightarrow \mathbb{R}^{n}$ e considere a equação diferencial

$$
\dot{x}(t)=f(t, x(t))
$$

com a condição inicial

$$
x\left(t_{0}\right)=x_{0} .
$$

Seguindo a terminologia de Coddington e Levinson (1955), diz-se que $\varphi$ é uma solução de 2 no sentido estendido em $I$, se $\varphi$ for uma solução no sentido de Carathéodory em $I$.

Teorema 3.2 (Coddington e Levinson (1955)). Seja $f$ definida em $D=\left\{(t, x) \in \mathbb{R} \times \mathbb{R}^{n}:\left|t-t_{0}\right| \leq\right.$ a e $\left.\left|x-x_{0}\right| \leq b\right\}$, e suponha que $f$ seja mensurável em $t$ para cada $x$ fixo e contínua em $x$ para cada $t$ fixo. Se existir uma função Lebesgue integrável $p$ no intervalo $\left|t-t_{0}\right| \leq a$ tal que

$$
|f(t, x)| \leq p(t) \quad((t, x) \in D)
$$

então existe uma solução $\varphi$ de 2 no sentido estendido em algum intervalo $\left|t-t_{0}\right| \leq \beta$, com $\beta>0$, satisfazendo $\varphi\left(t_{0}\right)=x_{0}$.

\subsection{Soluções de Filippov e de Krasovskii}

Soluções de Filippov foram abordadas, por exemplo, por Spraker e Biles (1996), Ceragioli (2002), Hajek (1979) e por Filippov (1988). Já as soluções de Krasovskii foram abordadas, por exemplo, por Ceragioli (2002), Hajek (1979) e por Hu (1991). Neste trabalho, supõe-se que o campo vetorial $f: \mathbb{R}^{n} \rightarrow \mathbb{R}^{n}$ seja Lebesgue mensurável e localmente limitado para definir as soluções de Filippov e de Krasovskii para o problema de valor inicial 1 .

As noções de soluções de Filippov e de Krasovskii para o problema 1 consiste em substituir a equação diferencial dada em 1 por uma inclusão diferencial adequada

$$
\dot{x}(t) \in F(x(t))
$$

onde $F$ é uma dada multifunção, ou seja, uma aplicação que associa a cada $x \in \mathbb{R}^{n}$ um subconjunto de $\mathbb{R}^{n}$. Diremos que uma multifunção $F$ definida em $\Omega \subset \mathbb{R}^{n}$ é fechada, compacta, convexa ou nãovazia para significar que sua imagem $F(x)$ satisfaz a propriedade requerida para cada $x \in \Omega$. Uma solução ordinária da inclusão diferencial 3 será qualquer função absolutamente contínua $x:[0, T] \rightarrow$ 
$\mathbb{R}^{n}$ satisfazendo $\dot{x}(t) \in F(x(t))$ para q.t.p. $t \in[0, T]$. Soluções de Filippov para o problema 1 são as soluções ordinárias da inclusão diferencial 3 obedecendo $x(0)=x_{0}$, onde

$$
F(x)=F_{F}(x)=\bigcap_{\delta>0} \bigcap_{\mu(N)=0} \overline{c o}\{f(\mathcal{B}(x, \delta) \backslash N)\}
$$

enquanto as soluções de Krasovskii do problema 1 são as soluções ordinárias da inclusão diferencial 3 obedecendo $x(0)=x_{0}$, sendo

$$
F(x)=F_{K}(x)=\bigcap_{\delta>0} \overline{c o}\{f(\mathcal{B}(x, \delta))\}
$$

onde $\mu$ é a medida de Lebesgue de $\mathbb{R}^{n}, \overline{c o}$ denota o fecho do envoltório convexo, e $\mathcal{B}(x, r)$ é a bola aberta de raio $r$ centrada em $x$. Como $F_{F}(x) \subset F_{K}(x)$, toda solução de Filippov é uma solução de Krasovskii. Para analisar propriedades da multifunção $F_{F}$, temos também a proposição dada a seguir.

Proposição 3.3 (Aubin e Cellina (1984)). Seja $f: \Omega \subset \mathbb{R}^{n} \rightarrow \mathbb{R}^{n}$, onde $\Omega$ é um conjunto aberto. Suponha que a função $f$ seja localmente limitada. Seja a multifunção $\phi$ definida por

$$
\phi(x)=\bigcap_{\delta>0} \bigcap_{\mu(N)=0} \overline{c o}\{f((\mathcal{B}(x, \delta) \cap \Omega) \backslash N)\} .
$$

Então

(i) a multifunção $\phi$ é não-vazia, convexa e semicontínua superior,

(ii) se $f$ é contínua em $x, \phi(x)=\{f(x)\}$.

Suponha também que $f$ seja mensurável em $\Omega$. Então

(iii) $f(x) \in \phi(x)$ para q.t.p. $x \in \Omega$.

A partir de Aubin e Cellina (1984), abordamos a existência de soluções para a inclusão diferencial 3 quando a multifunção $F$ for semicontínua superior. Dessa forma, introduzimos primeiro o conceito de aplicação localmente compacta e então enunciamos o resultado de existência de soluções dado em Aubin e Cellina (1984).

Definição 3.4. Dizemos que uma aplicação $\phi$ é localmente compacta se para cada ponto em Dom $(\phi)$ existir uma vizinhança que é aplicada em um subconjunto compacto.

Se $K$ é um subconjunto fechado e convexo, define-se $m(K)$ como o elemento de $K$ com a menor norma. 
Teorema 3.5 (Aubin e Cellina (1984)). Seja $X$ um espaço de Hilbert, $\Omega \subset \mathbb{R} \times X$ um subconjunto aberto contendo $\left(0, x_{0}\right)$. Seja $F$ uma multifunção semicontínua superior de $\Omega$ nos subconjuntos convexos fechados não-vazios de $X$. Suponha que a aplicação $(t, x) \mapsto m(F(t, x))$ seja localmente compacta. Então existe $T>0$ e uma função absolutamente contínua $x(\cdot)$ definida em $[0, T]$, uma solução para a inclusão diferencial

$$
\dot{x}(t) \in F(t, x(t)), x(0)=x_{0} .
$$

Com relação ao problema de valor inicial 1, como estamos supondo que o campo vetorial $f: \mathbb{R}^{n} \rightarrow \mathbb{R}^{n}$ seja Lebesgue mensurável e localmente limitado, então a multifunção $F_{F}(x)$ é semicontínua superior, localmente limitada, compacta e convexa. O mesmo vale para a multifunção $F_{K}(x)$. Do Teorema 3.5 o problema 1 tem uma solução de Filippov (e, portanto, uma solução de Krasovskii) em algum intervalo $[0, c]$.

\subsection{Soluções de Sentis}

Soluções de Sentis foram tratadas, por exemplo, por Bacciotti (2004), Bacciotti (2005), Sentis (1978) e por Krbec (1981). Soluções de Sentis para o problema 1 serão definidas como $g$-soluções da inclusão diferencial 3. Assim, $g$-soluções são definidas abaixo. Aqui nós também assumimos que o campo vetorial $f: \mathbb{R}^{n} \rightarrow \mathbb{R}^{n}$ seja Lebesgue mensurável e localmente limitado.

De acordo com Sentis (1978) substituímos a equação diferencial dada em 1 por uma inclusão diferencial 3 onde

$$
F(x)=F_{S}(x)=\bigcap_{\delta>0} \bigcap_{\mu(N)=0} \overline{\{f(\mathcal{B}(x, \delta) \backslash N)\}}
$$

A multifunção $F_{S}(x)$ é semicontínua superior, localmente limitada e compacta (mas em geral nãoconvexa). Como observado por Bacciotti (2004), temos também que $F_{F}(x)=c o\left\{F_{S}(x)\right\}$. Dessa forma, se $f$ for uma função contínua, então $F_{F}(x)=F_{S}(x)=\{f(x)\}$.

Seja $x_{0}$ um ponto fixo de $\mathbb{R}^{n}$. Dado $m \in \mathbb{N}$, considere uma partição de $[0, T]$

$$
0=t_{m, 0}<t_{m, 1}<\cdots<t_{m, k_{m}-1}<t_{m, k_{m}}=T
$$

onde $k_{m}$ é algum inteiro positivo, e seja

$$
l_{m}=\max \left\{t_{m, i+1}-t_{m, i}, i=0, \ldots, k_{m}-1\right\} .
$$


Então, para cada $i=0, \ldots, k_{m}-1$ tome $\varepsilon_{m, i} \in \mathbb{R}^{n}$ e construa uma função linear por partes $\psi_{m}(t)$ no intervalo $[0, T]$ satisfazendo $\psi_{m}\left(t_{m, 0}\right)=x_{0}$, e

$$
\psi_{m}\left(t_{m, i+1}\right)=\psi_{m}\left(t_{m, i}\right)+v_{m, i}\left(t_{m, i+1}-t_{m, i}\right)+\varepsilon_{m, i}
$$

onde $v_{m, i}$ é um elemento arbitrário em $F\left(\psi_{m}\left(t_{m, i}\right)\right)$. A função $\psi_{m}(t)$ será chamada de aproximação poligonal.

Definição 3.6. Uma função $\varphi:[0, T] \rightarrow \mathbb{R}^{n}$ é uma $g$-solução da inclusão diferencial[3 se para cada $\sigma>0$ existe um inteiro $m$ e uma aproximação poligonal obedecendo

$$
\left\|\varphi(t)-\psi_{m}(t)\right\|<\sigma \quad \forall t \in[0, T]
$$

$0<l_{m}<\sigma$ e $0 \leq \sum_{i=0}^{k_{m}-1}\left\|\varepsilon_{m, i}\right\|<\sigma$.

Por construção, $\varphi(0)=x_{0}$. Sentis (1978) prova que se $F(x)$ for uma multifunção semicontínua superior, localmente limitada e compacta, então para cada $x_{0}$ existem $c>0$ e uma $g$ solução $\varphi:[0, c] \rightarrow \mathbb{R}^{n}$ com $\varphi(0)=x_{0}$. Sentis (1978) também prova que soluções ordinárias da inclusão diferencial 3, quando elas existem, são $g$-soluções. Outras propriedades relativas a $g$-soluções podem ser encontradas em Sentis (1978).

Dizemos que uma função $\varphi(t)$ é uma solução de Sentis do problema 1 se ela for uma $g$ solução da inclusão diferencial $3 \operatorname{com} F(x)=F_{S}(x)$.

Proposição 3.7 (Sentis (1978)). Suponha que a função $f: \mathbb{R}^{n} \rightarrow \mathbb{R}^{n}$ seja Lebesgue mensurável e localmente limitada. Então cada solução de Sentis do problema 1 é uma solução de Filippov.

\section{Relações entre $\mathcal{C}\left(f, x_{0}\right)$ e $\mathcal{S}\left(f, x_{0}\right)$}

Nesta seção são obtidos os resultados principais do trabalho. Tais resultados são afirmados no Teorema 4.1, Teorema 4.5 e Teorema 4.10. Os referidos teoremas foram obtidos a partir de analogias com resultados considerados por Hu (1991) e por Spraker e Biles (1996).

Inicialmente, observamos que em geral uma solução de Carathéodory não é uma solução de Sentis. Para ver isso, considere a função $f: \mathbb{R} \rightarrow \mathbb{R}$ dada por

$$
f(x)= \begin{cases}0, & x=0 \\ 1, & x \neq 0\end{cases}
$$


e seja $x_{0}=0$. Neste caso $x(t) \equiv t$ é uma solução de Sentis do problema 1 . Como $x(t) \equiv t$ é a única solução de Filippov neste caso, então $\mathcal{S}(f, 0)=\{t\}$. Assim, como $x_{1}(t) \equiv 0$ é uma solução de Carathéodory do problema 1 , temos que $x_{1}$ não é uma solução de Sentis, ou seja, $x_{1} \notin \mathcal{S}(f, 0)$. Observamos também que em geral uma solução de Sentis não é uma solução de Carathéodory. De fato, se a função $f: \mathbb{R} \rightarrow \mathbb{R}$ é dada por

$$
f(x)= \begin{cases}1, & x=0 \\ 0, & x \neq 0\end{cases}
$$

e $x_{0}=0$, então $\mathcal{S}(f, 0)=\{0\}$. Como $x_{1}(t) \equiv 0$ não é uma solução de Carathéodory do problema 1. segue que $x_{1} \notin \mathcal{C}(f, 0)$. Neste caso $\mathcal{C}(f, 0)=\emptyset$. Entretanto, destacamos que $\mathcal{C}\left(f, x_{0}\right)=\mathcal{S}\left(f, x_{0}\right)$ quando $f: \mathbb{R}^{n} \rightarrow \mathbb{R}^{n}$ for uma função contínua.

No Teorema 4.1 abaixo estabelecemos condições necessárias para que $\mathcal{C}\left(f, x_{0}\right)=\mathcal{S}\left(f, x_{0}\right)$. O Teorema 4.1 estabelece uma analogia com o Teorema 1 dado em Spraker e Biles (1996). Teorema 1 dado em Spraker e Biles (1996) fornece uma condição necessária para a igualdade entre os conjuntos de soluções de Carathéodory e de Filippov.

Teorema 4.1. Seja $f: \mathbb{R}^{n} \rightarrow \mathbb{R}^{n}$ Lebesgue mensurável e localmente limitada. Então, a condição

$$
\forall x_{0} \in \mathbb{R}^{n}, \quad \mathcal{C}\left(f, x_{0}\right)=\mathcal{S}\left(f, x_{0}\right)
$$

implica as seguintes condições:

(i) $\forall x \in \mathbb{R}^{n}, \quad f(x)=0$ implica que $0 \in F_{F}(x)$.

(ii) $\forall x \in \mathbb{R}^{n}, \quad 0 \in F_{S}(x)$ implica que $f(x)=0$.

Prova. (i) Seja $x_{1} \in \mathbb{R}^{n}$ de modo que $f\left(x_{1}\right)=0$. Seja $x(t) \equiv x_{1}$. Então, $\dot{x}(t) \equiv 0$ em $[0, T]$. Assim, $x \in \mathcal{C}\left(f, x_{1}\right)$ e então $x \in \mathcal{S}\left(f, x_{1}\right)$, já que por hipótese $\mathcal{C}\left(f, x_{1}\right)=\mathcal{S}\left(f, x_{1}\right)$. Como $x \in \mathcal{S}\left(f, x_{1}\right)$ implica que $x$ é uma solução de Filippov, temos que $0=\dot{x}(t) \in F_{F}(x(t))=F_{F}\left(x_{1}\right)$ para q.t.p. $t \in[0, T]$, e dessa forma $0 \in F_{F}\left(x_{1}\right)$.

(ii) Seja agora $x_{1} \in \mathbb{R}^{n}$ tal que $0 \in F_{S}\left(x_{1}\right)$. Se $x(t) \equiv x_{1}$, então $x \in \mathcal{S}\left(f, x_{1}\right)$ e $\dot{x}(t) \equiv 0$ em $[0, T]$. Como $\mathcal{C}\left(f, x_{1}\right)=\mathcal{S}\left(f, x_{1}\right)$ por hipótese, concluímos que $x \in \mathcal{C}\left(f, x_{1}\right)$. Temos então que $0=\dot{x}(t)=f(x(t))=f\left(x_{1}\right)$ para q.t.p. $t \in[0, T]$, e assim $f\left(x_{1}\right)=0$. 
Destacamos que as condições (i) e (ii) do Teorema 4.1 não são suficientes para a igualdade dos conjuntos $\mathcal{C}\left(f, x_{0}\right)$ e $\mathcal{S}\left(f, x_{0}\right)$. Por exemplo, a função $f: \mathbb{R} \rightarrow \mathbb{R}$ dada por

$$
f(x)= \begin{cases}1, & x>0 \\ 0, & x=0 \\ -1, & x<0\end{cases}
$$

satisfaz a condição (i), e no entanto $\mathcal{C}(f, 0)=\{t,-t, 0\}$ e $\mathcal{S}(f, 0)=\{t,-t\}$ (como pode ser visto em Sentis (1978)). Por outro lado, a função $f: \mathbb{R} \rightarrow \mathbb{R}$ dada por

$$
f(x)= \begin{cases}0, & x=0 \\ 1, & x \neq 0\end{cases}
$$

satisfaz a condição (ii), mas $\mathcal{C}(f, 0)=\{t, 0\}$ e $\mathcal{S}(f, 0)=\{t\}$.

Entre os teoremas principais estudados por Hu (1991) estão o Teorema A e o Teorema B (afirmados em Hu (1991)). Por completeza e facilidade de leitura, abaixo enunciamos o Teorema A (como Teorema 4.2) e o Teorema B (como Teorema 4.3). Denotaremos por $\mathcal{K}\left(f, x_{0}\right)$ o conjunto de soluções de Krasovskii para o problema 1.

Teorema 4.2. Se $f: \mathbb{R}^{n} \rightarrow \mathbb{R}^{n}$ é contínua em $\mathbb{R}^{n}$ exceto em um subconjunto enumerável $\sigma$, então $\mathcal{C}\left(f, x_{0}\right)=\mathcal{K}\left(f, x_{0}\right)$ para quaisquer $x_{0} \in \mathbb{R}^{n} \Leftrightarrow x \in \sigma e 0 \in F_{K}(x)$ implica que $f(x)=0$.

Teorema 4.3. Sejam $\sigma$ um conjunto de Borel em $\mathbb{R}$ com $\mu(\sigma)=0$ e $f: \mathbb{R} \rightarrow \mathbb{R}$ uma função contínua exceto em $\sigma$. Se $x \in \sigma$ e $0 \in F_{K}(x) \Rightarrow f(x)=0$, então

$$
\mathcal{K}\left(f, x_{0}\right)=\mathcal{C}\left(f, x_{0}\right) .
$$

Convém observar que nos Teoremas 4.2 e 4.3, a função $f$ é limitada e satisfaz a seguinte condição,

$$
|f(x)| \leq M(1+|x|) \quad \forall x \in \mathbb{R}^{n}
$$

para algum $M>0$. Destacamos também a relação $\mathcal{C}\left(f, x_{0}\right) \subset \mathcal{K}\left(f, x_{0}\right)$, já que $f(x) \in F_{K}(x)$ para todo $x \in \mathbb{R}^{n}$.

No Teorema 4.5 estabelecemos condições necessárias e suficientes para que $\mathcal{S}\left(f, x_{0}\right) \subset$ $\mathcal{C}\left(f, x_{0}\right)$. De modo que fica estabelecida uma analogia entre o Teorema 4.5 e o Teorema 4.2. Para a obtenção do Teorema 4.5 necessitamos do Lema 4.4 abaixo.

Lema 4.4 (Hobson (1957)). Se $I \subset \mathbb{R}$ não for enumerável, então I pode ser expresso como

$$
I=I_{1} \cup I_{2}
$$


onde $I_{1}$ é enumerável, e, para qualquer $x \in I_{2}$,

$$
x \in \overline{\left\{y \in I_{2}: y>x\right\}} \cap \overline{\left\{y \in I_{2}: y<x\right\}}
$$

Teorema 4.5. Seja $\sigma=\left\{x_{i}: i \geq 1\right\} \subset \mathbb{R}^{n}$. Suponha que $f: \mathbb{R}^{n} \rightarrow \mathbb{R}^{n}$ seja contínua em todo $x \in \mathbb{R}^{n} \backslash \sigma$. Suponha também que $f$ seja localmente limitada. Então:

(i) $x \in \sigma$ e $0 \in F_{F}(x)$ implica que $f(x)=0 \Rightarrow \mathcal{S}\left(f, x_{0}\right) \subset \mathcal{C}\left(f, x_{0}\right)$ para quaisquer $x_{0} \in \mathbb{R}^{n}$.

(ii) $\mathcal{S}\left(f, x_{0}\right) \subset \mathcal{C}\left(f, x_{0}\right)$ para quaisquer $x_{0} \in \mathbb{R}^{n} \Rightarrow x \in \sigma$ e $0 \in F_{S}(x)$ implica que $f(x)=0$.

Prova. (i) Dada $x \in \mathcal{S}\left(f, x_{0}\right)$, defina $I_{i}=\left\{t \in[0, T]: x(t)=x_{i}\right\}$ e $I=\bigcup_{i=1}^{\infty} I_{i}$. Para qualquer $i$ fixado, se $\mu\left(I_{i}\right) \neq 0$, então do Lema 4.4 temos que $\dot{x}(t)=0$ para q.t.p. $t \in I_{i}$. Logo $0 \in F_{F}(x(t))$ para q.t.p. $t \in I_{i}$, já que $x$ também é uma solução de Filippov. Assim, $\dot{x}(t)=f(x(t))$ para q.t.p. $t \in I_{i}$. Consequentemente, $\dot{x}(t)=f(x(t))$ para q.t.p. $t \in I$. Do item (ii) da Proposição 3.3 segue que $F_{F}(x(t))=\{f(x(t))\}$ para todo $t \in[0, T] \backslash I$. Portanto, $\dot{x}(t)=f(x(t))$ para q.t.p. $t \in[0, T]$, e assim $x \in \mathcal{C}\left(f, x_{0}\right)$. Isto prova que $\mathcal{S}\left(f, x_{0}\right) \subset \mathcal{C}\left(f, x_{0}\right)$.

(ii) Suponha que $0 \in F_{S}\left(x_{i}\right)$ e no entanto $f\left(x_{i}\right) \neq 0$ para algum $i \geq 1$. Defina $x(t) \equiv x_{i}$ em $[0, T]$, então $x \in \mathcal{S}\left(f, x_{i}\right)$ mas $x \notin \mathcal{C}\left(f, x_{i}\right)$. Logo, $\mathcal{S}\left(f, x_{i}\right) \not \subset \mathcal{C}\left(f, x_{i}\right)$ e obtemos uma contradição com a hipótese de que $\mathcal{S}\left(f, x_{0}\right) \subset \mathcal{C}\left(f, x_{0}\right)$ para quaisquer $x_{0} \in \mathbb{R}^{n}$. Portanto $f\left(x_{i}\right)=0$ e a prova está completa.

No Teorema 4.10 obtemos uma condição suficiente para que $\mathcal{S}\left(f, x_{0}\right) \subset \mathcal{C}\left(f, x_{0}\right)$. Assim, no Teorema 4.10 é obtida uma analogia com o Teorema 4.3. Para a obtenção do Teorema 4.10, utilizamos o Lema 4.6, Teorema 4.7 e o Lema 4.8, como enunciados abaixo. Denotaremos por $\mathcal{F}\left(f, x_{0}\right)$ o conjunto de soluções de Filippov para o problema 1.

Usaremos o Lema 3.6 dado em Hu (1991) (e enunciado abaixo como Lema 4.6) para a prova do Teorema 4.7 .

Lema 4.6. Sejam $y:[0, T] \rightarrow \mathbb{R}$ uma função absolutamente contínua, $g: y([0, T]) \rightarrow \mathbb{R}$ mensurável, $S \subset[0, T]$ mensurável. Suponha que $h(t)=g(y(t)) \dot{y}(t)$ seja integrável em $[0, T]$. Então:

(i) $\int_{y(a)}^{y(b)} g d s=\int_{a}^{b} h d t$ se $S=[a, b]$

(ii) $\int_{y(S)} g d s=\int_{S} h d t$ se $y$ for crescente,

(iii) $\int_{y(S)} g d s \leq \int_{S} g(y(t))|\dot{y}(t)| d t$ se $g \geq 0$ em $y([0, T])$. 
Teorema 4.7. Seja $\sigma$ quaisquer conjunto de Borel em $\mathbb{R} \operatorname{com} \mu(\sigma)=0$. Suponha que $f: \mathbb{R} \rightarrow \mathbb{R}$ seja contínua em $\mathbb{R}$ exceto em $\sigma$, e

$$
x \in \sigma \quad e \quad 0 \in F_{F}(x) \Rightarrow f(x)=0 .
$$

Então, qualquer $x$ monótona de $\mathcal{F}\left(f, x_{0}\right)$ também está em $\mathcal{C}\left(f, x_{0}\right)$.

Prova. Se $S=\left\{x^{-1}(\sigma)\right\}$, então $S$ é mensurável. Seja $x$ monótona em $\mathcal{F}\left(f, x_{0}\right)$ e considere $g=1$ e $y=x$ no item (ii) do Lema 4.6. Logo,

$$
0=\mu(\sigma)=\int_{\sigma} d s=\int_{S} \dot{x}(t) d t .
$$

Como $\dot{x}(t) \geq 0$ para q.t.p. se $x$ for crescente, ou $\dot{x}(t) \leq 0$ para q.t.p. se $x$ for decrescente, temos que $\dot{x}(t)=0$ para q.t.p. $t \in S$. Assim, $\dot{x}(t)=f(x(t))$ para q.t.p. $t \in S$, e então $\dot{x}(t)=f(x(t))$ para q.t.p. $t \in[0, T]$. Portanto, $x \in \mathcal{C}\left(f, x_{0}\right)$.

Lema 4.8. Suponha que $f: \mathbb{R} \rightarrow \mathbb{R}$ seja contínua exceto em um subconjunto $\sigma=\sigma_{1}+\sigma_{2}$ de $\mathbb{R}$, onde $\sigma_{1}$ é um conjunto de medida zero mas $\sigma_{2}$ é arbitrário (pode não ser mensurável). Além disso, suponha que

$$
x \in \sigma_{1} \quad e \quad 0 \in F_{F}(x) \Rightarrow f(x)=0
$$

$e$

$$
x \in \sigma_{2} \Rightarrow 0 \notin F_{F}(x) .
$$

Então quaisquer $x(t)$ de $\mathcal{F}\left(f, x_{0}\right)$ é monótona.

O Lema 4.8 pode ser obtido de modo análogo ao Lema 3.10 dado em Hu (1991) e enunciado abaixo como Lema 4.9 .

Lema 4.9. Suponha que $f: \mathbb{R} \rightarrow \mathbb{R}$ seja contínua exceto em um subconjunto $\sigma=\sigma_{1}+\sigma_{2}$ de $\mathbb{R}$, onde $\sigma_{1}$ é um conjunto de medida zero mas $\sigma_{2}$ é arbitrário (pode não ser mensurável). Suponha também que

$$
x \in \sigma_{1} \quad e \quad 0 \in F_{K}(x) \Rightarrow f(x)=0
$$

$e$

$$
x \in \sigma_{2} \Rightarrow 0 \notin F_{K}(x) .
$$

Então quaisquer $x(t)$ de $\mathcal{K}\left(f, x_{0}\right)$ é monótona.

Finalmente, enunciamos e provamos a seguir o Teorema 4.10 . 
Teorema 4.10. Seja $\sigma$ um conjunto de Borel em $\mathbb{R}$ com $\mu(\sigma)=0$. Suponha que $f: \mathbb{R} \rightarrow \mathbb{R}$ seja contínua em $\mathbb{R}$ exceto em $\sigma$, localmente limitada e

$$
x \in \sigma \quad e \quad 0 \in F_{F}(x) \Rightarrow f(x)=0 .
$$

Então, $\mathcal{S}\left(f, x_{0}\right) \subset \mathcal{C}\left(f, x_{0}\right)$.

Prova. Seja $x \in \mathcal{S}\left(f, x_{0}\right)$. Logo, $x \in \mathcal{F}\left(f, x_{0}\right)$ e do Lema 4.8 segue que $x(t)$ é monótona. Assim, do Teorema 4.7 concluímos que $x \in \mathcal{C}\left(f, x_{0}\right)$. Portanto, $\mathcal{S}\left(f, x_{0}\right) \subset \mathcal{C}\left(f, x_{0}\right)$ e a prova está completa.

\section{Conclusões}

O presente artigo contribui para a literatura de equações diferenciais descontínuas, de modo que foram comparados os conjuntos de soluções de Carathéodory e de Sentis do problema de valor inicial 1. Assim, a partir de resultados estabelecidos por Spraker e Biles (1996) e por Hu (1991), são estabelecidos aqui resultados análogos para relações de inclusão entre os conjuntos de soluções de Carathéodory e de Sentis.

\section{Referências}

AUBIN, J.-P.; CELLINA, A. Differential inclusions. Berlin: Springer-Verlag, 1984.

BACCIOTTI, A. Generalized solutions of differential inclusions and stability. Italian Journal of Pure and Applied Mathematics, v. 17, p. 183-192, 2005.

BACCIOTTI, A. Some remarks on generalized solutions of discontinuous differential equations. International Journal of Pure and Applied Mathematics, v. 10, n. 3, p. 257-266, 2004.

CERAGIOLI, F. Some remarks on stabilization by means of discontinuous feedbacks. Systems \& Control Letters, v. 45, n. 4, p. 271-281, 2002.

CODDINGTON, E. A.; LEVINSON, N. Theory of ordinary differential equations. New York: McGraw-Hill, 1955.

FILIPPOV, A. F. Differential equations with discontinuous righthand sides. Dordrecht: Kluwer, 1988.

HAJEK, O. Discontinuous differential equations. v. I. Journal of Differential Equations, v. 32, p. 149-170, 1979.

HALE, J. K. Ordinary differential equations. Huntington: Robert E. Krieger Publishing Co., 1980. HOBSON, E. W. The theory of functions of a real variable. v. I, New York: Dover, 1957. 
HU, S. Differential equations with discontinuous right-hand sides. Journal of Mathematical Analysis and Applications, v. 154, n. 2, p. 377-390, 1991.

KRBEC, P. On nonparasit generalized solutions of differential relations. Časopis Pro Pěstování Matematiky, v. 106, p. 368-372, 1981.

ROYDEN, H. L. Real analysis. New York: The Macmillan Co., 1963.

SENTIS, R. Équations différentielles à second membre mesurable. Unione Matematica Italiana. Bollettino. B. Serie V, v. 15, n. 3, p. 724-742, 1978.

SMIRNOV, G. V. Introduction to the theory of differential inclusions. Providence: American Mathematical Society, 2002.

SPRAKER, J. S.; BILES, D. C. A comparison of the Carathéodory and Filippov solution sets. Journal of Mathematical Analysis and Applications, v. 198, n. 2, p. 571-580, 1996. 